\title{
Up to 5 years outcomes following intraoperative radiotherapy for early breast cancer
}

\author{
Laurence Bauwens Patas d'Illiers ${ }^{1}$, Frédéric Lafay ${ }^{1}$, Christelle Faure ${ }^{2}$, Sophie Klingler ${ }^{2}$, Elodie Pleynet ${ }^{3}$, Séverine \\ Racadot $^{1}$ \\ ${ }^{1}$ Radiotherapy Department, ${ }^{2}$ Surgical Department, ${ }^{3}$ UGBDI, Centre Léon Bérard, 28 rue Laennec, 69008 Lyon, France \\ Contributions: (I) Conception and design: LB d'Illiers, S Racadot; (II) Administrative support: E Pleynet, S Racadot; (III) Provision of study materials \\ or patients: LB d'Illiers, F Lafay, C Faure, S Klingler, S Racadot; (IV) Collection and assembly of data: LB d'Illiers, E Pleynet, S Racadot; (V) Data \\ analysis and interpretation: LB d'Illiers, E Pleynet, S Racadot; (VI) Manuscript All authors; (VII) Final approval of manuscript: All authors. \\ Correspondence to: Laurence Bauwens Patas d'Illiers. Radiotherapy Department, Centre Léon Bérard, 28 Promenade Léa et Napoléon Bullukian, \\ 69008 Lyon, France; 5 quai Marechal Joffre, 69002 Lyon, France. Email: laurencedilliers@gmail.com.
}

Background: Whole breast radiation therapy (WBRT) is the standard of care for early breast cancer after lumpectomy, but it has side effects. For elderly women with good prognostic cancer, intra-operative radiotherapy (IORT) is an alternative. We report our results with IORT.

Methods: We performed a retrospective cohort study to observe clinical outcomes after IORT, between 2012 and 2017. Patients had inclusion criteria for RIOP INCA (French national institute of cancer) (group A) or for TARGIT E (group B).

Results: Ninety women were included (91 tumors). The mean age was 73.1 years (range, 56.0-87.0 years) and $95.6 \%$ of tumors were under $20 \mathrm{~mm}$. After a median follow-up of 27.4 months (range, 3.0-51.0 months), overall survival was $100 \%$, with two local recurrences out of the 84 tumors followed at least 3 months (2.4\%). After IORT, $34.1 \%$ of patients had adjuvant WBRT; 70.2\% of tumors had fibrosis at least once in the followup, $47.6 \%$ had cystosteatonecrosis, $25.8 \%$ had breast pain grades $1-2$, $6 \%$ had over grade 2 toxicities, with no grade 4 , and $94.0 \%$ had good to very good subjective global aspect.

Conclusions: IORT is a good alternative to WBRT when ASTRO's guidelines indications are respected, with promising results, but need carry on following up with patients.

Keywords: Breast; neoplasm; radiotherapy; adjuvant

Submitted Apr 23, 2018. Accepted for publication Jun 07, 2018.

doi: $10.21037 /$ tcr.2018.06.07

View this article at: http://dx.doi.org/10.21037/tcr.2018.06.07

\section{Introduction}

Breast conserving surgery (BCS) and adjuvant whole breast radiation therapy (WBRT) is the standard of care for early breast cancer (1). American societies recommend omission of WBRT in women older than 70 years old (yo) with stage I, ER + breast cancer who plan to receive hormone therapy (2). Long-term prospective studies are still ongoing. Classical WBRT is a long treatment [usually 50 Gray (Gy) in daily fractions for 5 consecutive weeks with an external beam boost of 10 to the tumor bed] and has acute side effects and long-term toxicities.
An ipsilateral breast tumor recurrence occurs most often (85\%) in the tumor bed (within $2 \mathrm{~cm}$ of lumpectomy) (3). Furthermore, accelerated partial-breast irradiation (APBI) is a technique that can decrease treatment time and give a better quality of life by potential reduction in treatment toxicity. Hence, the European and American Societies for Radiation Oncology made recommendations for selected patients for APBI after breast-conserving surgery $(4,5)$. For the European Society, APBI can be proposed if women are over 50 yo with a tumor (size under $3 \mathrm{~cm}$ ), whereas for the American Society APBI can be proposed for women over 60 yo with a tumor size under $2 \mathrm{~cm}$. According to 
both Societies, it is necessary to have a favorable histology profile: verified invasive-ductal breast cancer, Scarf Bloom Richardson (SBR) grade $\leq 2$, Erb2-negative, hormone receptor-positive, node-negative. Multiple techniques can be used for APBI, including accelerator with or without intensity modulated radiotherapy (6), interstitial brachytherapy $(7,8)$, mammosite $(9,10)$ and intra-operative radiotherapy (IORT) with either electrons or low dose $\mathrm{X}$-rays $(11,12)$. These techniques have large differences in dose rate and dose distribution and are not strictly comparable. Therefore, we will report our results with IORT only with Intrabeam device.

This study aims to report acute and late toxicities, cosmetic outcome, local and distant control after IORT using $\mathrm{kV} \mathrm{X}$-rays in a monocentric French center.

\section{Methods}

We performed a descriptive retrospective exhaustive cohort study in our center (Léon Bérard Oncology Center, Lyon, France) to observe clinical outcomes after IORT in terms of local and distant control, acute and chronic toxicities, complementary radiotherapy and cosmetic results, between April 2012 and September 2017.

\section{Population}

All the patients who received IORT in our center from the beginning were includable, from January 2012 to August 2017.

At the beginning, IORT was performed in our center through two studies: RIOP for INCA and TARGIT E. RIOP study was a French randomized multicentric medicoeconomical study managed by INCA in 2011, which compared IORT versus WBRT. The aim of this study was to evaluate the cost of the two techniques. TARGIT $\mathrm{E}$ was a multicentric European study for elderly women (over 70 yo) who were treated with IORT (Intrabeam system) (13). The purpose of this single-arm trial was to investigate the efficacy of a single IORT treatment in elderly low-risk patients.

As these studies finished, we continued to offer IORT to patients who had inclusion criteria for RIOP INCA. Therefore, in our study, we separated patients at inclusion into two groups: patients with inclusion criteria for RIOP INCA (in the protocol, in the arm IORT only, and nonprotocol) in group A and patients with inclusion criteria for Targit E in group B. Inclusion criteria for group A were: menopausal women aged 55 and over, with no history of breast cancer, BRCA-wild type, presenting unifocal, $\leq 20 \mathrm{~mm}$ in size (clinically and by ultrasound), grade $\mathrm{SBR} \leq 2$, Erb2-negative, hormone receptor-positive, node-negative, histologically verified invasive-ductal breast cancer. Inclusion criteria for group B were: women aged 70 and over, BRCA-wild type, presenting unifocal (mammography and ultrasound), $\leq 35 \mathrm{~mm}$ in size, node-negative, without clinical signs of distant metastases or lymph vessels invasion, histologically verified invasive-ductal breast cancer.

\section{Data collection}

Patients were included and the first data were collected at the moment of IORT, and then outcomes were retrospectively collected in August 2017, using a database "Access" with CNIL (Information and Freedom National Committee) approval on 2012/11/14.

\section{Treatment}

IORT with Intrabeam device was delivered during BCS to the tumor bed after primary histological analysis of margins and sentinel nodes. Dose was $20 \mathrm{~Gy}$ at the applicator surface, with low energy $\mathrm{X}$-rays $(50 \mathrm{kV})$. The size of the spherical applicator was decided by the surgeon and the radiation oncologist according to the size of the tumor bed and was correlated to the duration of radiation. About half of the patients received antibiotics during the surgery (14).

After definite pathological results, adjuvant treatments were done according to a multidisciplinary staff. Complementary WBRT without boost on tumor bed was recommended in case of risk factors on the final results. In group A, risk criteria were: embolus, ganglion invasion, lobular or extensive intraductal component histology, insufficient margins $(<1 \mathrm{~mm})$, grade SBR 3, multifocal tumor, size larger than required by the study $(>2 \mathrm{~cm})$. In Group B, they were: extensive intraductal component, lymph vessels invasion, multifocality/-centricity, larger diameter $(>3.5 \mathrm{~cm})$, other histology or too small free resection margins $(<2 \mathrm{~mm})$. We delivered $50 \mathrm{~Gy}$ in 25 fractions to the whole breast in group A and 46 Gy in 23 fractions in Group B (or 50 Gy in 25 fractions in the breast and the nodes area in case of $\mathrm{pN}+$ ), following instructions of these trials. In case of large breast size, the fractionation could be changed to 50.4 Gy in 28 fractions at the discretion of the clinical physician. We performed WBRT including irradiation of the lymphatic drainage areas with $50 \mathrm{~Gy}$ in $\geq \mathrm{pN} 1$ situations. 


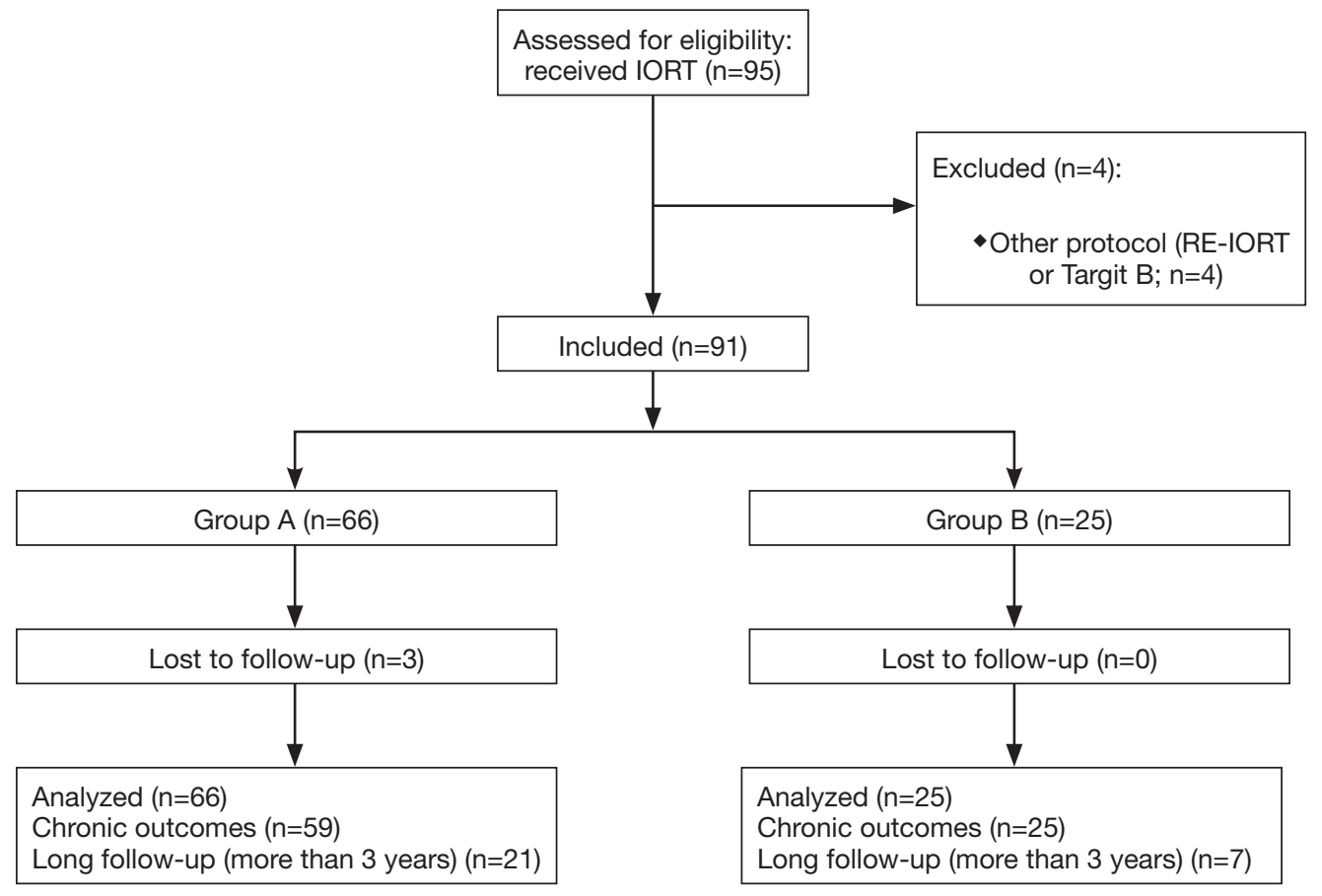

Figure 1 Flowchart.

\section{Outcomes}

Acute toxicities were defined by toxicities occurring at day 15 after IORT or within 3 months from IORT; they were reported at inclusion and graded using Common Terminology Criteria for Adverse Events scale v4 (CTCAEv4) (15). Late toxicities were reported retrospectively for patients with more than 3 months of follow-up, and were reported retrospectively using medical records, at 4-6 months, 1 year and then every year until September 2017; they were graded using modified Late Effects of Normal Tissues-Subjective, Objective, Management, Analytic (LENT-SOMA) scale (16-18). Cystosteatonecrosis was identified radiologically (mammography or ultrasound). Pain was evaluated using modified LENT-SOMA scale. Local and distant recurrence was reported retrospectively at the date of the analysis, and the date of recurrence was the date of pathological confirmation. The physicians and patients reported separately the cosmetic outcomes at day 15 , at 6 months and then every year, using a 5 items grade, on consistency, sensitivity, breast and mammillary symmetry and global aspect. Final cosmetic results are defined as the cosmetic result at last evaluation by each patient. We evaluated chronic toxicities and cosmetic results in the long follow- up group (over 3 years of follow-up) to evaluate longterm toxicities, separating patients treated with IORT and complementary WBRT or IORT only.

\section{Results}

\section{Participant flow and recruitment}

Ninety-four patients (95 tumors) received IORT by Intrabeam device in our center between April 2012 and August 2017. One patient (group A) had bilateral breast cancer and received IORT in each breast. Four were performed in other protocols (TARGIT-B and RE-IORT01) with other inclusion criteria and tumor characteristics and were not analyzed (Figure 1). RE-IORT01 (Intraoperative Radiotherapy after Local Recurrence in Breast Cancer) is a multicentric French protocol which aims to determine the feasibility of reirradiation with IORT (19). TARGIT B is a European multicentric randomized protocol that compares IORT boost versus external radiotherapy boost (20). We analyzed outcomes for 90 patients, meaning 91 tumors, 66 in group A and 25 in group B, as shown on Figure 1.

Median follow-up after IORT was 27.4 months (range, 3-51 months). Three patients were lost to follow-up (no follow-up for 18 months at the date of the study), 
and 7 had less than 3 months of follow-up, so they didn't have chronic toxicities and cosmetic outcome evaluation. Twenty-eight tumors $(30.8 \%)$ had a long follow-up (over 3 years).

\section{Baseline data}

Patient characteristics are summarized in Table 1. Average age was 73.1 yo (range, 56.0-87.0 yo) and was comparable between two groups. Ten tumors $(11.0 \%)$ had neoplastic history (4 had history of contralateral breast cancer, 5 had other cancer history, see details in Table 1). There were $87 \mathrm{~T} 1$ tumors (from TNM breast cancer staging) (95.6\%), $50(54.9 \%)$ with size $\leq 10 \mathrm{~mm}$ and $37(40.7 \%)$ measuring 10 to $20 \mathrm{~mm}$. There was only $1(1.1 \%)$ tumor over $30 \mathrm{~mm}$, and no tumor over $50 \mathrm{~mm}$ (T3). Probe size was 20 to $45 \mathrm{~mm}$, $83.5 \%$ of the population had probe size between 30 and $40 \mathrm{~mm}$, and average duration of the application was 24.3 minutes (range, 11.0-40.0 minutes).

All the tumors had invasive component, and 59 of them (64.8\%) had in-situ component. The main histology type was invasive ductal carcinoma (IDC) (83 tumors, 91.2\%). The other tumors' pathologies were: lobular, mixt (ductal and lobular), papillary and colloid.

On the initial biopsy, only one tumor (1.1\%) was hormone receptor-negative, and all were Erb2-negative, in immunohistochemistry (IHC), or after fluorescence in-situ hybridization (FISH) check. Ultimately, 87 tumors (95.6\%) received adjuvant hormone therapy, and 1 (1.1\%) adjuvant anti-Erb2 therapy (trastuzumab). Four tumors (4.4\%) had criteria for adjuvant chemotherapy and received docetaxel and cyclophosphamide.

As inclusion criteria were different, patients' characteristics seemed to be slightly different between two groups. There was one tumor (1.5\%) T2 (range, 20-50 mm) in group A vs. 3 (12.0\%) in group B, with 1 (4.0\%) over $30 \mathrm{~mm}$. Sixty-two tumors (93.9\%) were ductal in group A vs. $21(84.0 \%)$ only in group B, with $16 \%$ of other pathology. Four tumors (6.1\%) had adjuvant chemotherapy in group A, none in group B, and $64(97.0 \%)$ had adjuvant hormone therapy in group A, vs. 23 (92.0\%) in group B. Finally, median follow-up was 22.1 months in group A vs. 39.8 months in group B.

\section{Local recurrence rate and overall survival}

Overall survival was $100 \%$. Two local recurrences out of 84 tumors $(2.4 \%)$ were reported, one in each group. Among long follow-up group, these two recurrences were $7.1 \%$ of the group.

The first one (group A) occurred after 15 months. The first biopsy showed an 8-mm IDC, grade SBR 2, positive hormone-receptor, in a 71-yo patient, allowing IORT according to RIOP INCA criteria. The final pathology showed a 10-mm IDC, positive hormone-receptor, Erb2 positive, grade SBR 3, pT1bN0, with rare ductal in-situ carcinoma, sentinel node negative, without embolus or perinerve sheating. The patient had adjuvant chemotherapy (taxol) and trastuzumab for one year, and hormonal therapy, but did not have complementary radiotherapy. No radiation oncologist (specialized in Intrabeam) was present at the multidisciplinary staff and unfortunately adjuvant WBRT was not proposed for this patient. Recurrence happened on same localization (left intern quadrants junction) with an 8-mm IDC, positive estrogen-receptor, Erb2 positive, grade SBR 3, without in-situ carcinoma, embolus or perineural invasion. She had second lumpectomy and axillary node dissection, which was negative, WBRT and another hormone therapy. She has now 36 months of follow-up and no local or distant recurrence.

The second recurrence (group B) occurred after 33 months. The first biopsy showed a 7-mm IDC, grade SBR 3, hormone-receptor and Erb2 negative, in a 70-yo patient, allowing IORT according to Targit E criteria. Final pathology showed a $7-\mathrm{mm}$ IDC, with high grade insitu carcinoma with inframillimetric external margin, grade SBR 3, hormone-receptor and Erb2 negative, sentinel node negative, pT1bN0. The patient refused adjuvant chemotherapy, and didn't have complementary WBRT, according to Targit E protocol. Recurrence happened on same localization (left internal quadrants junction, on the lumpectomy scarf), with a 13-mm IDC, grade SBR 3, hormone-receptor and Erb2 negative. She refused every second treatment proposal and is still on follow-up at 42 months without local or distant metastasis.

\section{Complementary radiotherapy}

Thirty-one tumors had WBRT after IORT (34.1\%), 22 in group A (33.3\%), 9 in group B (36.0\%) (Table 2). The major indications were: ganglion invasion $(45.2 \%)$ which was more frequently micrometastasis $(32.3 \%)$, insufficient margins $(32.3 \%)$, and lobular histology $(12.9 \%)$. Other indications were SBR grade $3(9.7 \%)$, multifocal tumor (6.5\%), size (6.5\%), embolus (3.2\%), and $1(3.2 \%)$ had family background of ovarian and breast cancer before 50 yo. 
Table 1 Patients' and tumors' characteristics

\begin{tabular}{|c|c|c|c|}
\hline Characteristics & $\begin{array}{c}\text { Total } \\
(\mathrm{N}=91 \\
\text { tumors })\end{array}$ & $\begin{array}{c}\text { Group } \\
\text { A (N=66 } \\
\text { tumors) }\end{array}$ & $\begin{array}{c}\text { Group } \\
\mathrm{B}(\mathrm{N}=25 \\
\text { tumors) }\end{array}$ \\
\hline \multicolumn{4}{|l|}{ Age (years) } \\
\hline Average & 73.1 & 71.8 & 75.8 \\
\hline Min & 56.0 & 56.0 & 70.0 \\
\hline Max & 87.0 & 87.0 & 87.0 \\
\hline \multicolumn{4}{|c|}{ Neoplastic history $^{\dagger}, \mathrm{N}(\%)$} \\
\hline No & $81(89.0)$ & $58(87.9)$ & $23(92.0)$ \\
\hline Yes & $10(11.0)$ & $8(12.1)$ & $2(8.0)$ \\
\hline \multicolumn{4}{|l|}{ T stage, $\mathrm{N}(\%)$} \\
\hline TO & 0 & 0 & 0 \\
\hline $\mathrm{T} 1$ & 87 (95.6) & $65(98.5)$ & $22(88.0)$ \\
\hline $\mathrm{T} 2$ & $4(4.4)$ & $1(1.5)$ & $3(12.0)$ \\
\hline T3 & 0 & 0 & 0 \\
\hline \multicolumn{4}{|c|}{ Tumor size (mm), N (\%) } \\
\hline$\leq 10$ & $50(54.9)$ & $39(59.1)$ & $11(44.0)$ \\
\hline $10-20$ & $37(40.7)$ & $26(39.4)$ & $11(44.0)$ \\
\hline $20-30$ & $3(3.3)$ & $1(1.5)$ & $2(8.0)$ \\
\hline$>30$ & $1(1.1)$ & 0 & $1(4.0)$ \\
\hline \multicolumn{4}{|c|}{ Probe size $(\mathrm{mm}), \mathrm{N}(\%)$} \\
\hline 20 & $1(1.1)$ & $1(1.5)$ & 0 \\
\hline 25 & $4(4.4)$ & $4(6.1)$ & 0 \\
\hline 30 & $16(17.6)$ & $12(18.2)$ & $4(16.0)$ \\
\hline 35 & $30(33.0)$ & $23(34.8)$ & 7 (28.0) \\
\hline 40 & $30(33.0)$ & $21(31.8)$ & $9(36.0)$ \\
\hline 45 & $10(11.1)$ & $5(7.6)$ & $5(20.0)$ \\
\hline \multicolumn{4}{|c|}{$\begin{array}{l}\text { Duration of the application } \\
\text { (min) }\end{array}$} \\
\hline Average & 24.3 & 23.8 & 25.6 \\
\hline Min & 11.0 & 11.0 & 17.0 \\
\hline Max & 40.0 & 40.0 & 37.0 \\
\hline \multicolumn{4}{|c|}{ Hormone receptor } \\
\hline+ & 90 (98.9) & 65 (98.5) & $\begin{array}{c}25 \\
(100.0)\end{array}$ \\
\hline- & $1(1.1)$ & $1(1.5)$ & 0 \\
\hline
\end{tabular}

Table 1 (continued)

\begin{tabular}{lccc}
\hline Characteristics & $\begin{array}{c}\text { Total } \\
(\mathrm{N}=91 \\
\text { tumors })\end{array}$ & $\begin{array}{c}\text { Group } \\
\mathrm{A}(\mathrm{N}=66 \\
\text { tumors })\end{array}$ & $\begin{array}{c}\text { Group } \\
\mathrm{B}(\mathrm{N}=25 \\
\text { tumors })\end{array}$ \\
\hline Erb2 & 0 & 0 & 0 \\
+ & 91 & 66 & 25 \\
- & $(100.0)$ & $(100.0)$ & $(100.0)$ \\
++ in IHC but $<6$ copies & $14(15.4)$ & $9(13.6)$ & $5(20.0)$ \\
in FISH & & &
\end{tabular}

Pathology

$\begin{array}{lccc}\text { Ductal } & 83(91.2) & 62(93.9) & 21(84.0) \\ \text { Lobular } & 4(4.4) & 3(4.5) & 1(4.0) \\ \text { Purely in situ } & 0 & 0 & 0 \\ \text { Mixt (ductal + lobular) } & 1(1.1) & 1(1.5) & 0 \\ \text { Papillary } & 2(2.2) & 0 & 2(8.0) \\ \text { Colloid (mucinous) } & 1(1.1) & 0 & 1(4.0)\end{array}$

In-situ component

$\begin{array}{llll}\text { Yes } & 59(64.8) & 42(63.6) & 17(68.0) \\ \text { No } & 32(35.2) & 24(36.4) & 8(32.0)\end{array}$

Adjuvant chemotherapy

$\begin{array}{cccc}\text { Yes } & 4(4.4) & 4(6.2) & 0 \\ \text { No } & 87(95.6) & 62(93.9) & 0\end{array}$

Adjuvant anti-Erb2 ${ }^{\ddagger}$ therapy (trastuzumab)

$\begin{array}{lccc}\text { Yes } & 1(1.1) & 1(1.5) & 0 \\ \text { No } & 90(98.9) & 65(98.5) & 0 \\ \text { Adjuvant hormone therapy } & & & \\ \text { Yes } & 87(95.6) & 64(97.0) & 23(92.0) \\ \text { No } & 4(4.4) & 2(3.0) & 2(8.0)\end{array}$

Follow-up (months)

\begin{tabular}{lccc} 
Median & 27.4 & 22.1 & 39.8 \\
Min & 3.0 & 3.0 & 31.0 \\
Max & 51.0 & 51.0 & 51.0 \\
\hline
\end{tabular}

No patient had neoadjuvant chemotherapy. ${ }^{\dagger}$, neoplastic history: 4 contralateral breast cancers, 5 other cancer histories: multiple myeloma, 2 thyroid cancer, 1 appendix neuro-endocrine cancer, 1 chondrosarcoma and melanoma; ${ }^{\ddagger}$, Erb2: Erb2 status was confirmed by FISH if IHC showed Erb2++ (15 patients). IHC, immunohistochemistry; FISH, fluorescence in-situ hybridization.

Table 1 (continued) 
Table 2 Complementary radiotherapy: indications and characteristics

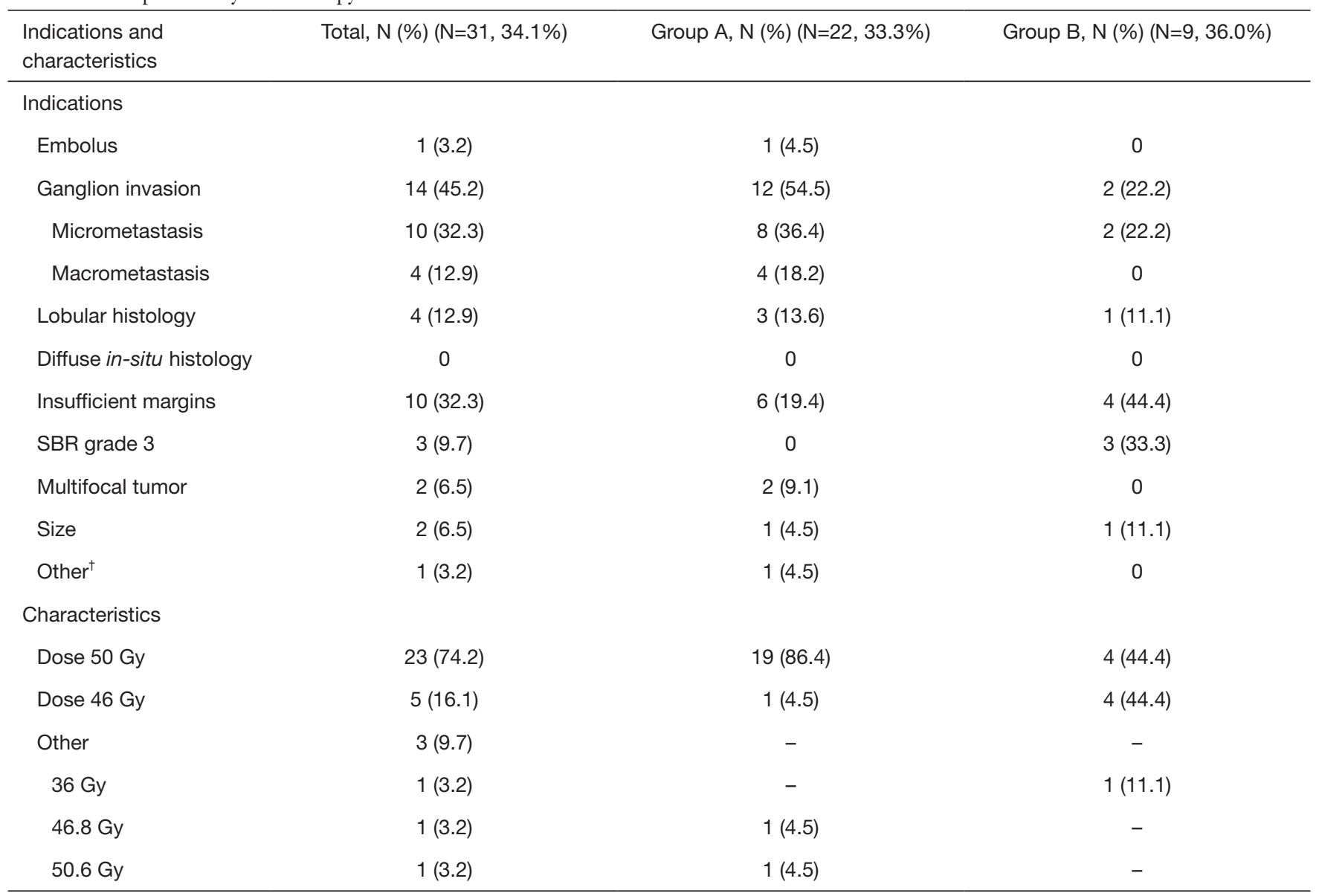

${ }^{\dagger}$, family background of ovarian and breast cancer before 50 years old. SBR, Scarf Bloom Richardson.

They received 50 Gy in 25 fractions for $74.2 \%$ of them on all breast, 46 Gy in 23 fractions for $16.1 \%$ of them and $9.7 \%$ of the entire population received another dose (50.6, 46.8 or $36 \mathrm{~Gy})$, at the appreciation of the physician or because of early stop for toxicities. Details are listed on Table 2.

\section{Acute toxicities}

Among 91 tumors with early follow-up, 15 (16.5\%) had infection, $15(16.5 \%)$ had seroma, and 23 over the 89 reported $(25.8 \%)$ had breast pain grades $1-2$. Eight-pointeight percent $(8.8 \%)$ had bleeding and $2.2 \%$ had wound dehiscence. We reported $8.8 \%$ grade $>2$ toxicity with no grade $4,3.3 \%$ grade 3 infection, $4.4 \%$ grade 3 seroma, and $1.1 \%$ grade 3 wound dehiscence. Acute toxicities are summarized in Table 3.

\section{Chronic toxicities}

Among 84 tumors with over 3 months of follow-up, $59(70.2 \%)$ had fibrosis at least once in the follow-up, $40(47.6 \%)$ cystosteatonecrosis, $39(46.4 \%)$ breast pain grades $1-2 ; 14.3 \%$ had retraction, $10.7 \%$ edema, $8.3 \%$ hyperpigmentation and $1.2 \%$ had telangiectasia and at least once. We had $6 \%$ grade $>2$ toxicities, with no grade $4,3.6 \%$ grade 3 fibrosis and $2.4 \%$ grade 3 edema. Chronic toxicities are summarized in Table 4.

\section{Cosmetic outcomes}

Cosmetic results were excellent with a good to very good subjective global aspect of 94\% (patients' evaluation). When we observed the answers in detail, we observed that 
Table 3 Acute toxicities after IORT

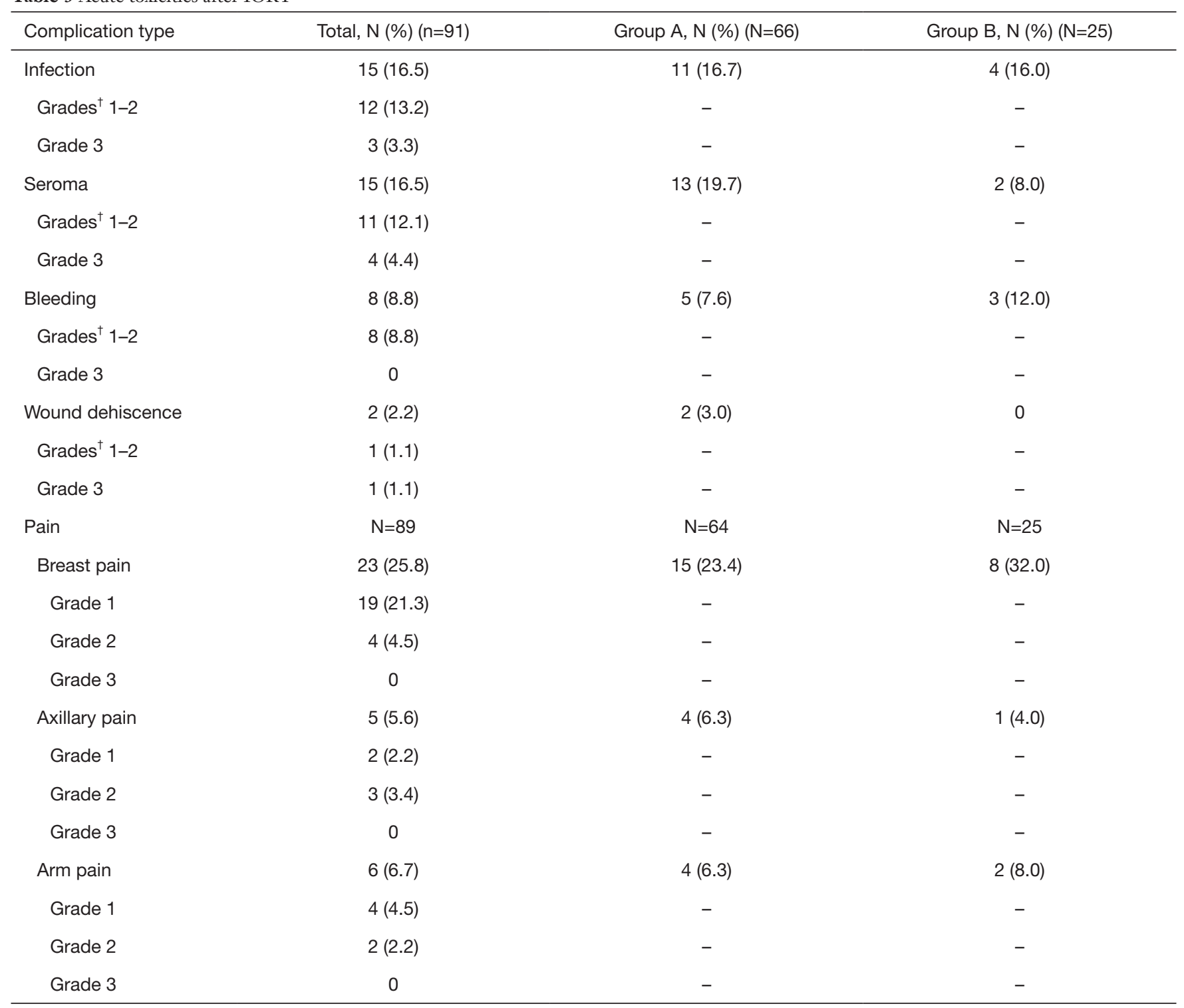

${ }^{\dagger}$, using CTCAEv4 scale. IORT, intra-operative radiotherapy.

three topics had more than $5 \%$ bad to very bad answers: sensitivity $(7.1 \%)$, breast symmetry $(7.1 \%)$ and nipple symmetry $(7.1 \%)$. Physicians' evaluations were notably similar, with $96.4 \%$ good to very good global aspect. Cosmetic results are summarized in Table 4.

\section{Results for long follow-up}

Long follow-up results are summarized in Table 5. Among 28 tumors with over 3 years of follow-up (7 in group A and 21 in group B), at their last follow-up, 19 (67.9\%) had fibrosis, 11 (39.3\%) had cystosteatonecrosis, 4 (14.3\%) had breast pain, 1 (3.6\%) had edema, and 1 (3.6\%) had hyperpigmentation; there was neither retraction nor telangiectasia. There was only $1(3.6 \%)$ grade $>2$ toxicity: one fibrosis grade 3 .

Among these tumors, 18 had only IORT and 10 had IORT and complementary WBRT. Patients who had IORT only seem to have had less fibrosis: 11 (61.1\%) vs. 8 (80.0\%), with no grade 3 fibrosis in IORT only group, less breast pain: 2 (11.1\%) vs. 2 (20.0\%), less edema: 0 vs. 1 (10.0\%), less hyperpigmentation: 0 vs. 1 (10.0\%). 
Table 4 Chronic toxicities after IORT

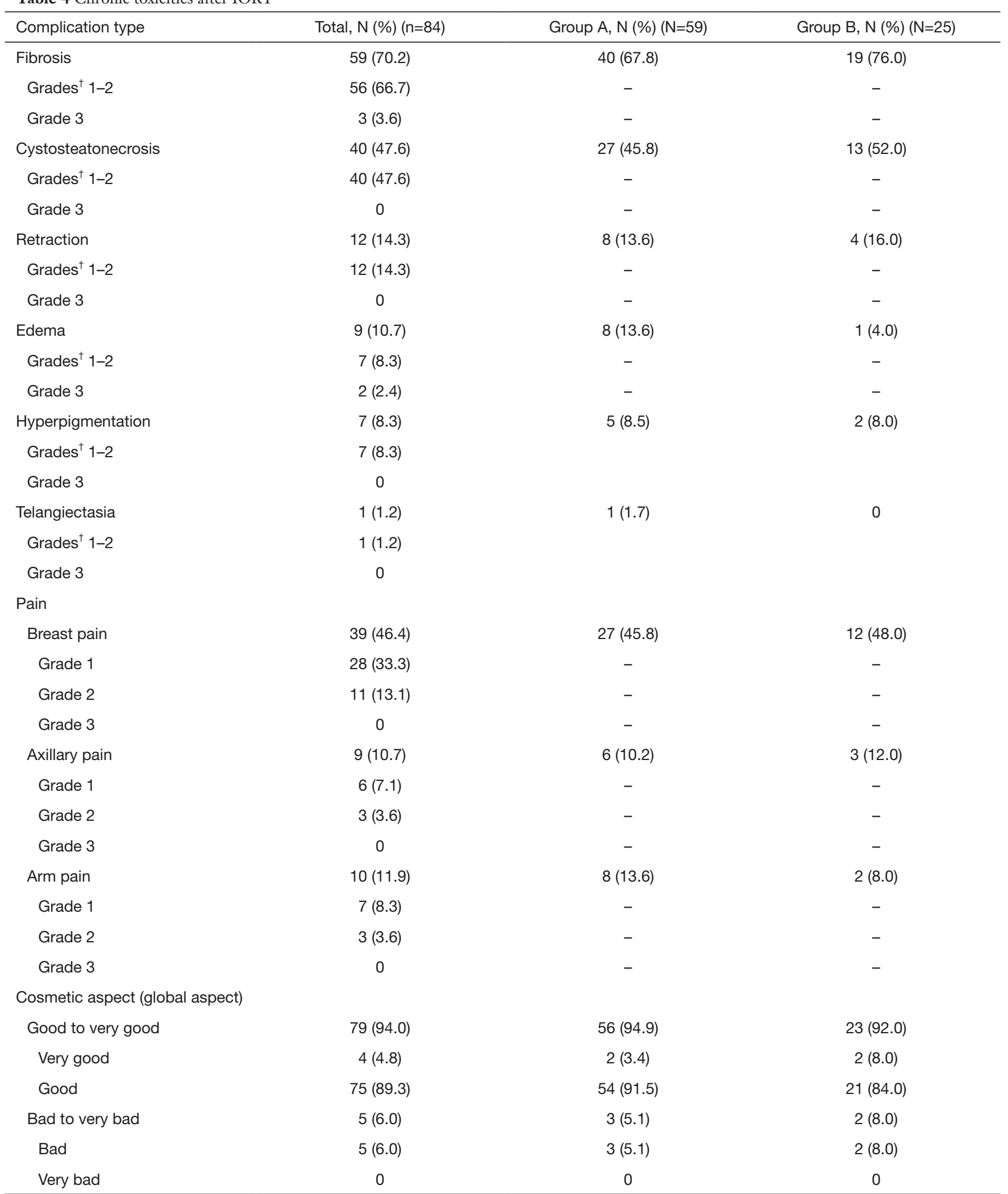

${ }^{\dagger}$, using LENT-SOMA scale. Incidence of toxicities occurring at least once in follow-up (LENT-SOMA scale, CTCAEv4 scale for pain), at least 3 months after IORT: 84 tumors with more than 3 months of follow-up. IORT, intra-operative radiotherapy. 
Table 5 Long-term toxicities after IORT

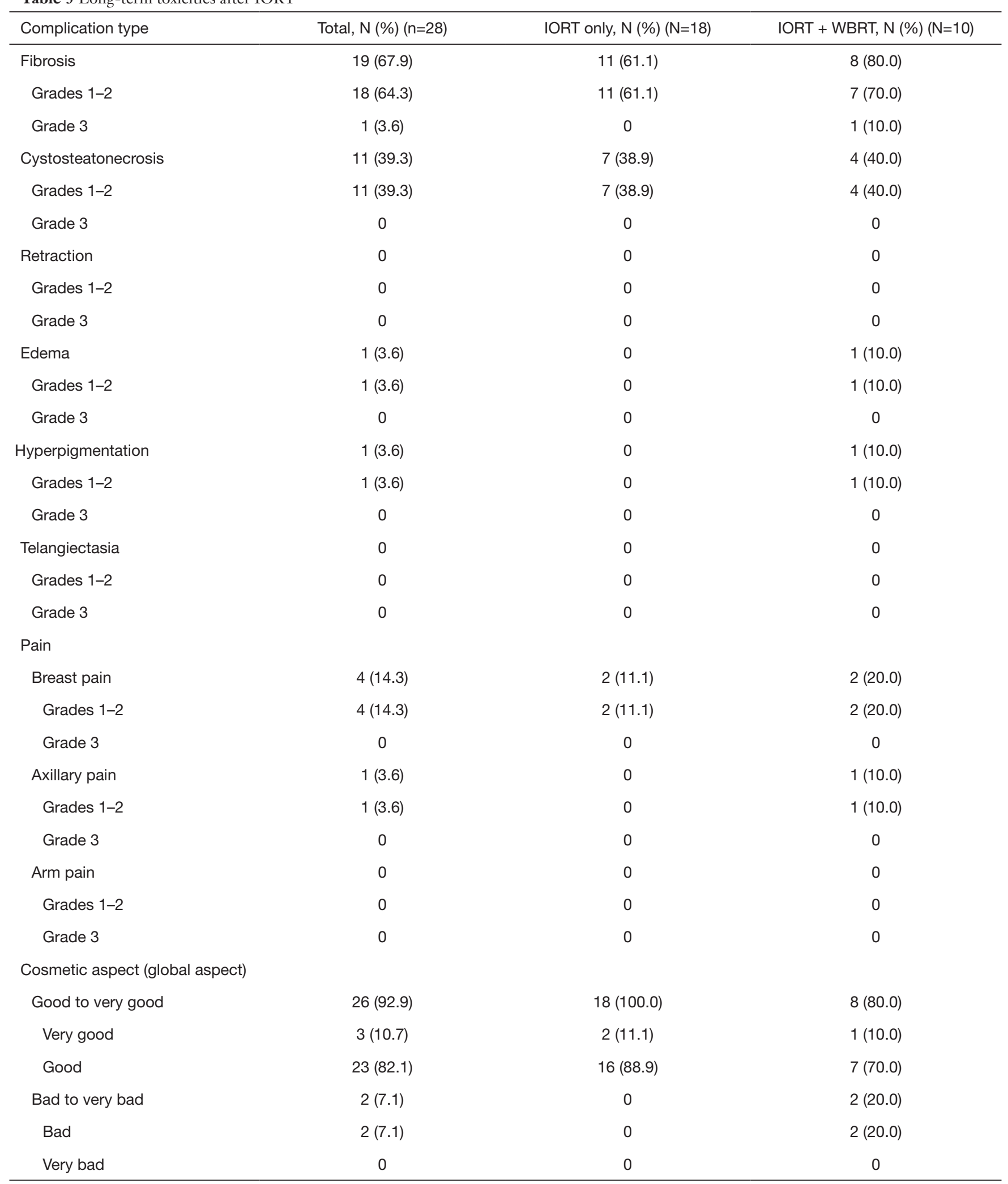

IORT, intra-operative radiotherapy; WBRT, whole breast radiation therapy. 
Regarding cosmetic aspect, 26 tumors (92.9\%) had good to very good global aspect. It seemed better in IORT only group than in IORT + WBRT group: $18(100.0 \%)$ vs. 8 $(80.0 \%)$ good to very good aspect.

\section{Discussion}

This study is one of the first studies reporting acute and late toxicities, cosmetic outcomes, and local and distant control after IORT using $\mathrm{kV} \mathrm{X}$-rays. Some studies focus on acute toxicities $(21,22)$, or on late toxicities (23). Vinh-Hung et al. reported their experience at Geneva University Hospitals (24) with 52 women receiving IORT treatment.

Our follow-up is short (median 27.4 months) but we followed 28 tumors $(30.8 \%)$ over 3 years. Two randomized phase III studies, ELIOT trial (25) and TARGIT-A trial $(26,27)$ have been developed regarding the use of IORT with results at a medium follow-up of 5.8 and 2.4 years respectively. In our study, local recurrence rate is $2.2 \%$. In TARGIT-A trial, local recurrence rate at 5 years in the IORT group was $3.3 \%$, while in ELIOT trial it was $4.4 \%$. In our study, two local recurrences occurred in the same localization 15 and 33 months after surgery and IORT. Both patients didn't have WBRT despite the SBR 3 grade in both recurrences. One patient had adjuvant chemotherapy with Herceptin and the other one refused chemotherapy.

In our study, $34.1 \%$ of the patients had adjuvant breast radiotherapy. Thirty-five percent of patients received postoperative radiotherapy in the Vinh-Hung study because of unfavorable histology (if the 2009 recommendations of the GEC-ESTRO were not validated in the postoperative pathological examination) (24). In the TARGIT-A trial, the rate of additional WBRT was only $21.6 \%$ in the prepathology stratum because of different criteria for WBRT (embolus and hormone receptor-negative were exclusion criteria both in our and in Vinh-Hung study) (27).

Acute toxicities were usual after BCS, with $16.5 \%$ infection, $16.5 \%$ seroma, and $25.8 \%$ breast pain grades 1-2: Heneghan et al. showed that after breast conservative surgery there was $11.8 \%$ infection (28). Severe acute toxicities were low: $3.3 \%$ of infection grade $3,4.4 \%$ of seroma grade 3 and $1.1 \%$ of wound dehiscence grade 3 . Our results are comparable to Key's study (23), which found $16 \%$ of delayed healing (over 1 month), with ulceration of the tumor bed in one patient out of three. In Key's study, $42 \%$ of patients had hematoseroma, $16.3 \%$ had erythema and $36 \%$ had grade 1 pain. In TARGIT A study, the severe acute toxicities were similar with $1 \%$ of hematoma needing surgical aspiration, $2.1 \%$ of seroma needing more than 3 aspirations, $1.8 \%$ of infections needing intravenous antibiotics or surgical intervention, $2.8 \%$ of skin breakdown or delayed wound healing (12).

Our chronic toxicity rate appears to be significant, but we reported the toxicity as present if it occurred at least once in the follow-up. That's why we also analyzed the results for the 28 long follow-up tumors (more than 3 months follow-up). The severe toxicities were low with $3.6 \%$ of fibrosis grade 3 . If we consider only the women who had IORT without WBRT, there was no fibrosis grade 3 and only $11 \%$ of breast pain (grade 1). In TARGIT A study, the results are available only for skin complications, which are less important in IORT group than WBRT (27). Our results are similar to Key's study (23), which reported $4.8 \%$ of grade 2 or higher toxicities with one grade 2 fibrosis and one grade 4 infection (resulting in breast ulceration). A majority of patients experienced grade 1 toxicities, with $46.3 \%$ grade 1 fibrosis and $41.5 \%$ grade 1 skin retraction; $14.6 \%$ of patients reported grade 1 breast pain, $7.3 \%$ of patients had grade 1 breast edema, $14.6 \%$ of patients had grade 1 telangiectasia, and $9.8 \%$ of patients had grade 1 pigmentation. In Deneve's study, after Intrabeam procedure for 42 patients, there was $11 \%$ of asymptomatic palpable seroma, $2 \%$ of scar retraction, $9.5 \%$ of noninfectious localized hyperemia (29).

Cosmetic results were excellent with $94.0 \%$ of good to very good global aspect. In Key's study, objective and subjective scores were very good (8.87 and 8.89 out of 10), vs. 6.96 and 6.51 in IORT + WBRT group (23).

\section{Conclusions}

Retrospective or prospective studies on accelerated partial breast irradiation (APBI) in adjuvant setting report promising results, both in terms of tolerance (saving healthy tissue) and local control ( $74 \%$ to $100 \%$ at 5 years).

IORT belongs to APBI techniques. It's important to respect ASTRO's guidelines with the size of tumor $(<2 \mathrm{~cm})$. The technique can be easily applied with minimal increase in operative length. The acute and late toxicities are low. It is important to inform patients that, in case of unfavorable histology, a WBRT must be done to ensure local control. WBRT is recommended to supplement IORT if there are adverse prognostic factors: tumor-free margin smaller than $1 \mathrm{~mm}$, extensive in-situ component, or unexpected invasive lobular carcinoma, positive nodes (micro or macro metastasis), extensive lymph vascular 
invasion or grade SBR 3.

Nevertheless, it is important to continue to follow up with patients to be sure it is a safe way of treatment.

\section{Acknowledgments}

The authors would like to thank MD Christian Carrie, MD Ronan Tanguy and MD Violaine Beneyton for revising the article and for general supervision, and Astrid d'Illiers for language editing assistance.

Funding: None.

\section{Footnote}

Conflicts of Interest: All authors have completed the ICMJE uniform disclosure form (available at http://dx.doi. org/10.21037/tcr.2018.06.07). The authors have no conflicts of interest to declare.

Ethical Statement: The authors are accountable for all aspects of the work in ensuring that questions related to the accuracy or integrity of any part of the work are appropriately investigated and resolved. The study was conducted in accordance with the Declaration of Helsinki (as revised in 2013). The written informed consent was waived due to the retrospective nature of the study. The study was approved by the National Ethics Committee of CNIL (on 2012/11/14).

Open Access Statement: This is an Open Access article distributed in accordance with the Creative Commons Attribution-NonCommercial-NoDerivs 4.0 International License (CC BY-NC-ND 4.0), which permits the noncommercial replication and distribution of the article with the strict proviso that no changes or edits are made and the original work is properly cited (including links to both the formal publication through the relevant DOI and the license). See: https://creativecommons.org/licenses/by-nc-nd/4.0/.

\section{References}

1. Early Breast Cancer Trialists' Collaborative Group (EBCTCG), Darby S, McGale P, et al. Effect of radiotherapy after breast-conserving surgery on 10year recurrence and 15-year breast cancer death: metaanalysis of individual patient data for 10,801 women in 17 randomised trials. Lancet 2011;378:1707-16.

2. Horton JK, Jagsi R, Woodward WA, et al. Breast Cancer
Biology: Clinical Implications for Breast Radiation Therapy. Int J Radiat Oncol Biol Phys 2018;100:23-37.

3. Gage I, Recht A, Gelman R, et al. Long-term outcome following breast-conserving surgery and radiation therapy. Int J Radiat Oncol Biol Phys 1995;33:245-51.

4. Polgár C, Van Limbergen E, Pötter R, et al. Patient selection for accelerated partial-breast irradiation (APBI) after breast-conserving surgery: recommendations of the Groupe Européen de Curiethérapie-European Society for Therapeutic Radiology and Oncology (GEC-ESTRO) breast cancer working group based on clinical evidence (2009). Radiother Oncol 2010;94:264-73.

5. Smith BD, Arthur DW, Buchholz TA, et al. Accelerated partial breast irradiation consensus statement from the American Society for Radiation Oncology (ASTRO). Int J Radiat Oncol Biol Phys 2009;74:987-1001.

6. Valachis A, Mauri D, Polyzos NP, et al. Partial breast irradiation or whole breast radiotherapy for early breast cancer: a meta-analysis of randomized controlled trials. Breast J 2010;16:245-51.

7. Strnad V, Ott OJ, Hildebrandt G, et al. 5-year results of accelerated partial breast irradiation using sole interstitial multicatheter brachytherapy versus whole-breast irradiation with boost after breast-conserving surgery for low-risk invasive and in-situ carcinoma of the female breast: a randomised, phase 3, non-inferiority trial. Lancet 2016;387:229-38.

8. Kamrava M, Kuske RR, Anderson B, et al. Outcomes of Breast Cancer Patients Treated with Accelerated Partial Breast Irradiation Via Multicatheter Interstitial Brachytherapy: The Pooled Registry of Multicatheter Interstitial Sites (PROMIS) Experience. Ann Surg Oncol 2015;22 Suppl 3:S404-11.

9. Wallace M, Martinez A, Mitchell C, et al. Phase I/II study evaluating early tolerance in breast cancer patients undergoing accelerated partial breast irradiation treated with the mammosite balloon breast brachytherapy catheter using a 2-day dose schedule. Int J Radiat Oncol Biol Phys 2010;77:531-6.

10. Benitez PR, Keisch ME, Vicini F, et al. Five-year results: the initial clinical trial of MammoSite balloon brachytherapy for partial breast irradiation in early-stage breast cancer. Am J Surg 2007;194:456-62.

11. Veronesi U, Orecchia R, Luini A, et al. Full-dose intraoperative radiotherapy with electrons (ELIOT) during breast-conserving surgery: experience with 1246 cases. Ecancermedicalscience 2008;2:65.

12. Vaidya JS, Joseph DJ, Tobias JS, et al. Targeted 
intraoperative radiotherapy versus whole breast radiotherapy for breast cancer (TARGIT-A trial): an international, prospective, randomised, non-inferiority phase 3 trial. Lancet 2010;376:91-102.

13. Neumaier C, Elena S, Grit W, et al. TARGIT-E(lderly)-prospective phase II study of intraoperative radiotherapy (IORT) in elderly patients with small breast cancer. BMC Cancer 2012;12:171.

14. Williams NR, Pigott KH, Brew-Graves C, et al. Intraoperative radiotherapy for breast cancer. Gland Surg 2014;3:109-19.

15. Atkinson TM, Ryan SJ, Bennett AV, et al. The association between clinician-based common terminology criteria for adverse events (CTCAE) and patient-reported outcomes (PRO): a systematic review. Support Care Cancer 2016;24:3669-76.

16. Pavy JJ, Denekamp J, Letschert J, et al. EORTC Late Effects Working Group. Late effects toxicity scoring: the SOMA scale. Radiother Oncol 1995;35:11-5.

17. Rubin P, Constine LS, Fajardo LF, et al. RTOG Late Effects Working Group. Overview. Late Effects of Normal Tissues (LENT) scoring system. Int J Radiat Oncol Biol Phys 1995;31:1041-2.

18. Hoeller U, Tribius S, Kuhlmey A, et al. Increasing the rate of late toxicity by changing the score? A comparison of RTOG/EORTC and LENT/SOMA scores. Int J Radiat Oncol Biol Phys 2003;55:1013-8.

19. Intraoperative Radiotherapy After Local Recurrence in Breast Cancer (RE-IORT01). Available online: https:// clinicaltrials.gov/ct2/show/NCT02386371

20. A Comparison of Intra-operative Radiotherapy Boost with External Beam Radiotherapy Boost in Early Breast Cancer. (TARGIT-B). Available online: https://clinicaltrials.gov/ ct2/show/NCT01792726

21. Tuschy B, Berlit S, Romero S, et al. Clinical aspects of

Cite this article as: d'Illiers LB, Lafay F, Faure C, Klingler S, Pleynet E, Racadot S. Up to 5 years outcomes following intraoperative radiotherapy for early breast cancer. Transl Cancer Res 2018;7(4):859-870. doi: 10.21037/tcr.2018.06.07 intraoperative radiotherapy in early breast cancer: shortterm complications after IORT in women treated with low energy x-rays. Radiat Oncol 2013;8:95.

22. Zur M, Shai A, Leviov M, et al. Short-term complications of intra-operative radiotherapy for early breast cancer. J Surg Oncol 2016;113:370-3.

23. Key S, Miglierini P, Dupré PF, et al. Cosmetic Outcome and Chronic Breast Toxicity After Intraoperative Radiation Therapy (IORT) as a Single Modality or as a Boost Using the Intrabeam(®) Device: A Prospective Study. Ann Surg Oncol 2017;24:2547-55.

24. Vinh-Hung V, Nepote V, Rozenholc A, et al. First year experience with IORT for breast cancer at the Geneva University Hospitals. Transl Cancer Res 2014;3:65-73.

25. Veronesi U, Orecchia R, Maisonneuve P, et al. Intraoperative radiotherapy versus external radiotherapy for early breast cancer (ELIOT): a randomised controlled equivalence trial. Lancet Oncol 2013;14:1269-77.

26. Sperk E, Welzel G, Keller A, et al. Late radiation toxicity after intraoperative radiotherapy (IORT) for breast cancer: results from the randomized phase III trial TARGIT A. Breast Cancer Res Treat 2012;135:253-60.

27. Vaidya JS, Wenz F, Bulsara M, et al. Risk-adapted targeted intraoperative radiotherapy versus wholebreast radiotherapy for breast cancer: 5-year results for local control and overall survival from the TARGIT-A randomised trial. Lancet 2014;383:603-13.

28. Heneghan HM, Prichard RS, Lyons R, et al. Quality of life after immediate breast reconstruction and skin-sparing mastectomy - a comparison with patients undergoing breast conserving surgery. Eur J Surg Oncol 2011;37:937-43.

29. Deneve JL, Hoefer RA Jr, Harris EE, et al. Accelerated partial breast irradiation: a review and description of an early North American surgical experience with the intrabeam delivery system. Cancer Control 2012;19:295-308. 